\title{
Estudio de la intención emprendedora de los alumnos de la Universidad Tecnológica El Retoño, México
}

\section{Study of the Entrepreneurial Intention of Students at the Technological University El Retoño, Mexico}

\author{
Magali Valdivia-Velasco \\ UNIVERSIDAD TECNOLÓGICA \\ El Retoño, México \\ magali.valdivia@utr.edu.mx \\ ORCID 0000-0001-9793-5109 \\ Graciela Coronado-Guzmán \\ Universidad TeCNOLÓGica \\ El Retoño, México \\ graciela.coronado@utr.edu.mx \\ ORCID 0000-0001-9225-9789
}

\author{
Humberto Martínez-Bautista \\ UnIVERSIDAD TECNOLÓGICA \\ El Retoño, México \\ humberto.martinez@cimat.mx \\ ORCID 0000-0001-6984-9430 \\ Aída Aguilera-Dávila \\ UNIVERSIDAD TECNOLÓGICA \\ El Retoño, México \\ aida.aguilera@utr.edu.mx \\ ORCID 0000-0002-7644-3241
}

\begin{abstract}
RESUMEN
El propósito de este estudio es medir la intención emprendedora de los alumnos de la Universidad Tecnológica El Retoño que cursaron el cuatrimestre mayo-agosto 2019, para ello se utilizó un instrumento diseñado, validado y aplicado por Saadin y Daskin en 2015, integrado por 25 reactivos estructurados en cuatro categorías: intención emprendedora, deseabilidad percibida, normas sociales y factibilidad percibida. La consistencia de cada una de las secciones del instrumento aplicado fue validada a través del alfa de Cronbach, correlaciones biseriales y la prueba estadística no paramétrica de correlación de Spearman. Utilizando el análisis de componentes principales, se construyó un Índice de Emprendimiento, el cual permitió medir la intención emprendedora y el contraste de las hipótesis. Se concluye que la intención emprendedora de los estudiantes de licenciatura e ingeniería sobresale de los alumnos de Técnico Superior Universitario y el de las carreras administrativas es el más alto de la institución.
\end{abstract}

Palabras clave: intención emprendedora, Universidad Tecnológica El Retoño, emprendimiento, componentes principales

\begin{abstract}
The purpose of this study is to know the entrepreneurial intention of the students at the Technological University El Retoño during the term May-August 2019. With this purpose, we used the instrument designed, validated, and used for Saadin and Dasking during the year 2015. It has 25 items integrated into four categories (entrepreneurial intention, perceived desirability, social norms, and perceived feasibility). The validation of consistency of each instrument's section was through Cronbach's alpha and the test of Spearman correlation. Using main components analysis, we built an Entrepreneurial Index, which allowed us to measure de entrepreneurial intention and to contrast hypotheses. We concluded the entrepreneurial intention of last terms' students and administrative students are the highest of the university.
\end{abstract}

Keywords: entrepreneurial intention, Technological University El Retoño, entrepreneurship, main components 


\section{INTRODUCCIÓN}

El emprendimiento es una tendencia actual que influye en la vida económica, social, política y cultural de la población de nuestro país. Bajo la premisa de que el emprendimiento se puede aprender en las aulas (Potishuk y Kratzer, 2017), su enseñanza se intensifica cada vez más en el ámbito de las universidades, como una respuesta emergente a diversas necesidades, principalmente del entorno económico y social. Según Mayer et al. (2019), el perfil de los estudiantes recién egresados debe corresponder con lo que demanda el mercado laboral, para afrontar las tasas altas de desempleo, los bajos salarios, la informalidad y la alta tasa de mortandad de los negocios de reciente creación, así como condiciones económicas cambiantes. Para la Universidad Tecnológica El Retońo, ubicada en el estado de Aguascalientes, el emprendimiento es un compromiso tanto a nivel regional como nacional e incluso internacional, ya que al ser la primera universidad pública bilingüe en México, a lo largo de su historia ha buscado que los alumnos de la institución aporten, más que su mano de obra, sus ideas innovadoras para desarrollar su capacidad emprendedora. Para determinar cuáles son las estrategias que la universidad debe establecer en materia de emprendimiento, se propone identificar la intención emprendedora (IE) de los alumnos a través de este estudio, para que, en un futuro cercano, ésta se materialice en la creación de nuevas empresas. El artículo está integrado de la siguiente manera: primero se presenta la fundamentación teórica del tema, en segundo lugar, el contexto del estudio y las hipótesis planteadas, donde se detalla la metodología empleada; posteriormente se exponen los resultados y la discusión para finalizar con las conclusiones del estudio.

El gobierno también es copartícipe de incentivar el emprendimiento, no sólo a través de la legislación, sino generando un adecuado soporte financiero y diseñando programas académicos que faciliten la educación en este rubro (Raposo y Paço, 2011; Fayolle, 2013); sin embargo, se debe señalar la brecha existente entre el emprendimiento que se enseńa y el que se practica. Según Hansemark (1998), en la enseñanza tradicional hay una transformación de conocimientos y habilidades, mientras que en la enseñanza del 
emprendimiento hay un cambio de actitudes y motivos, pues ahí se aprende a reconocer oportunidades y a generarlas, a crear nuevas ideas y a encontrar los recursos necesarios para materializarlas, ya que se desarrollan las habilidades para crear y administrar una nueva empresa, para pensar de forma creativa y crítica (Raposo y Paço, 2011). Este tipo de educación no sólo sirve para crear empresas, sino para convertir las inspiraciones en acciones en cualquier ámbito de la vida de los individuos. Sin embargo, en México el proceso no se ha desarrollado con la misma rapidez que en Estados Unidos, Canadá u otros países desarrollados (Mayer et al., 2019), y sigue habiendo evidencias de que las universidades siguen formando a los alumnos como empleados, en lugar de como empresarios. Valencia et al., 2012 y Mayer et al., 2019 seńalan tres requisitos para que una universidad fomente el emprendimiento en los estudiantes: 1) formar profesores y académicos en la creación de empresas (contando con el apoyo de incubadoras de negocios, programas de formación y currículos adecuados); 2) tener un modelo de formación emprendedora, y 3) analizar el desempeńo de la institución como generadora de profesionales dedicados a la investigación, al desarrollo de ideas innovadoras y a la creación de empresas.

Fomentando la educación del emprendimiento también se busca la reducción del número de los emprendedores por necesidad, e incentivando el de oportunidad (Kautonen et al., 2015), así como abatir el alto índice de fracasos en las empresas y aumentar la eficiencia y la innovación en la industria con el desarrollo de nuevas estrategias (Saadin y Daskin, 2015). Una forma de medir y predecir la capacidad de emprendimiento de un individuo es mediante el estudio de la intención emprendedora (IE).

La IE se puede definir como el estado de la mente que dirige la atención, la experiencia y la acción a desarrollar algún concepto de negocio (Bird, 1988). Para que surja una persona que quiera emprender es necesario que exista una oportunidad emprendedora y la intención y capacidad para aprovecharla, haciendo frente a la incertidumbre, al riesgo (Fayolle y Liñán, 2014, Kautonen et al., 2015) y al miedo al fracaso. Esto implica que la IE del individuo debe considerar variables individuales y ambientales (Contreras et al., 2017). Krueger et al. (2000) establecieron que, para estimular 
el desarrollo económico mediante la creación de nuevas empresas es necesario incrementar la percepción de viabilidad y deseabilidad en los individuos, lo cual propicia el incremento de la IE.

De acuerdo con Fayolle et al. (2014), existen tres modelos que sustentan el desarrollo de la IE, el de la Implementación de Ideas Emprendedoras (IIE) de Bird (1988), el Modelo del Evento Emprendedor (MEE) de Shapero y Sokol (1982), relacionado con los eventos emprendedores y el de la Teoría del Comportamiento Planificado (TCP) de Ajzen (1991).

La IIE plantea lo siguiente: 1) distingue entre emprendimiento y administración estratégica; 2) establece el impacto que tienen las intenciones emprendedoras en la dirección, sobrevivencia y crecimiento de una organización, consistentemente con las teorías de liderazgo, el desarrollo organizacional y la teoría de la organización, y 3) el planteamiento de que los emprendedores se basan en ideas y experiencias personales para desarrollar sus negocios, las cuales, con el tiempo, conforman la teoría del emprendimiento (Bird, 1988).

El MEE establece que la intención de crear una empresa depende de tres elementos: 1) el deseo de crearla, 2) la percepción de la viabilidad y 3) la percepción de la oportunidad (Krueger et al., 2000).

La TCP considera que la intención es un indicador del esfuerzo y la disposición para ejecutar un comportamiento cuando sea el momento y se dé la oportunidad adecuada. Los tres factores que lo explican son: 1) actitud hacia el comportamiento (evaluación personal favorable o desfavorable hacia dicho comportamiento); 2) normas subjetivas (percepción que se tiene en cuanto a la presión social de realizar o no una acción), y 3) control conductual percibido (la facilidad o dificultad para la realización del comportamiento con base en los obstáculos de experiencias pasadas). Segal et al. (2005) establecen una modificación al modelo de TCP, denominado Modelo de Intención Emprendedora (MIE), en el que se sustituye el factor de las normas subjetivas por el de tolerancia al riesgo, considerando que éste tiene gran incidencia en la IE. Por otro lado, Soria-Barreto et al. (2017) adicionan al modelo de Ajzen tres variables exógenas: el ambiente universitario, la educación del emprendimiento y la intención emprendedora previa.

La experiencia profesional bajo ciertas circunstancias tiene efectos positivos en la IE (Romero-Martínez y Milone, 2016) y, en otras, 
efectos negativos (Soria-Barreto et al., 2017). Algo similar sucede con lo establecido por Soria-Barreto et al. (2016a), donde se encontró que la IE disminuyó en individuos que descubrieron sus potenciales o asistieron a algún curso de emprendimiento.

En el estudio más reciente realizado por la Global Entrepreneurship Monitor (GEM) se encontró que los países de Latinoamérica y el Caribe presentan una mayor tasa de IE que de actividad empresarial total (GEM, 2018), lo cual es preocupante puesto que indica que, en cuanto a la medición de IE, los resultados son buenos, pero ésta no se está concretando o aterrizando en la generación de innovación o en la creación de nuevas empresas. En el estudio mencionado anteriormente, México no fue considerado, sólo se le incluyó para el índice del contexto nacional de emprendimiento donde, mediante el uso de 12 variables, se midió el ambiente del emprendimiento. El propósito de dicho índice fue investigar si las condiciones de una economía son favorables para el emprendimiento; México ocupó la posición 23 de 54 países considerados en dicho estudio, con un puntaje de 5.2, frente al más alto, que fue de 6.7. Cabe destacar que Guatemala tuvo la posición, 45 con un puntaje de 4.3. Este país sí fue considerado en el estudio de la GEM, donde estuvo evaluado con los índices más altos de actividad total de emprendimiento entre los países de Latinoamérica y el Caribe, seguido por Chile y Perú (GEM, 2018). Es, pues, necesario analizar por qué se ha gestado este cambio en el país, al extremo de ya no ser considerado parte del estudio de la GEM. Por ese motivo, un primer paso es medir la IE de los alumnos universitarios, determinar un punto de partida y establecer los programas necesarios para que se materialice, al paso de los ańos, en una actividad empresarial total que fomente no sólo el desarrollo económico regional, sino también el nacional y que eso sea el catalizador para fomentar el desarrollo social, familiar y cultural.

\section{CONTEXTO E HIPÓTESIS}

La Universidad Tecnológica El Retońo se fundó en 2012, como la primera universidad pública con el modelo Bilingüe Internacional Sustentable (BIS) en México. Está ubicada en el centro de la República mexicana, en el estado de Aguascalientes. Ofrece una doble titulación, pues los alumnos, al cursar seis cuatrimestres, pueden 
obtener el título de técnico superior universitario (TSU) y si así lo decidieran, pueden cursar cinco cuatrimestres más para obtener el grado de ingeniero o licenciado. Las carreras que se imparten a nivel técnico superior universitario son ocho, entre las que se encuentran: Administración Área Capital Humano, Desarrollo de Negocios Área Mercadotecnia, Mecatrónica Área Automatización, Mecatrónica Área Sistemas de Manufactura Flexible, Tecnologías de la Información Área Desarrollo de Software Multiplataforma, Tecnologías de la Información Área Infraestructura de Redes Digitales, Lengua Inglesa y Diseño Digital Área Animación. A nivel Licenciatura e Ingeniera se tienen las siguientes carreras: Ingeniería en Desarrollo e Innovación Empresarial, Ingeniería en Mecatrónica, Ingeniería en Tecnologías de la Información y Licenciatura en Gestión Institucional Educativa y Curricular. Cabe mencionar que los alumnos de la última generación de Ingeniería en Desarrollo e Innovación Empresarial fueron los del cuatrimestre mayo-agosto 2019, debido a que el plan de estudios de esta carrera se dividió en la Licenciatura en Gestión de Capital Humano y la Licenciatura en Innovación de Negocios y Mercadotecnia.

Debido a que el emprendedurismo es parte de las políticas de la institución, puesto que se considera una alternativa real de apoyo a la población estudiantil para superar el desempleo que se vive en la actualidad, el objetivo de este estudio es medir la IE de los estudiantes en el cuatrimestre mayo-agosto de 2019, para determinar cuál es la situación actual de la comunidad y, con base en ello, determinar las líneas de acción a seguir. Atendiendo las directrices del estudio se establecieron las siguientes hipótesis.

\section{Nivel de estudios}

De acuerdo con el estudio realizado por la GEM, en la mayoría de economías la edad en la que los individuos inician un negocio es entre los 25 y los 34 años o entre los 35 y los 44 años. La razón de ello es que poseen conocimientos y experiencia, pero aún no tienen un trabajo que les ofrezca beneficios atractivos como para no considerar viable el emprendimiento. Sin embargo, empiezan a surgir grupos de jóvenes que poseen la energía y el coraje suficiente para emprender, aunado con la capacitación que se les ha impartido desde edades 
tempranas en el interior de las escuelas, ejemplo de ello es el caso de Brasil (GEM, 2018).

Obschonka et al. (2010) encontraron que, incentivando el emprendimiento durante la adolescencia, se logra que durante la adultez esta actividad se vea como algo natural y seguro de que pueda suceder. En el estudio realizado por Contreras et al. (2017) con muestras de los países de Latinoamérica, ellos encontraron que los estudiantes que están próximos a egresar de la universidad muestran una mayor intención emprendedora que los que están iniciando sus estudios universitarios. Por otro lado, según el GEM, en países como Chile, Colombia, Ecuador, Perú y Venezuela los universitarios jóvenes tienen una alta IE. De acuerdo con lo anterior, se plantea la siguiente hipótesis de investigación:

$H_{1}$ : La IE de los alumnos que cursan los últimos cuatrimestres es mayor que la de los alumnos que cursan los primeros cuatrimestres.

\section{Tipo de carreras}

Hay varios estudios, entre los que se encuentra el de Dugassa (2012), quien establece que los alumnos de ingeniería tienen una menor IE que los que estudian alguna carrera en el área de negocios debido, principalmente, a la educación emprendedora recibida en dichas carreras: Debido a ello se establece la siguiente hipótesis de investigación y se considera su análisis en el resto de las carreras impartidas por la institución.

$\mathrm{H}_{2}$ : Los alumnos de las carreras administrativas tienen la IE más alta de la Universidad.

\section{METODOLOGÍA}

\section{Instrumento}

Se utilizó el instrumento diseñado y validado por Saadin y Daskin en 2015, cuya intención fue medir la IE en estudiantes de las carreras de hotelería en Malasia. Los reactivos del instrumento están agrupados en cuatro secciones: 1) intención emprendedora (enten- 
dida como los esfuerzos que se realizan para la creación de un negocio), 2) deseabilidad percibida (es el grado en que a una persona le es atractivo crear un negocio), 3) normas sociales (es la influencia social que condiciona los pensamientos del individuos con respecto al emprendimiento), y 4) factibilidad percibida (este concepto está relacionado con la autoeficacia, la cual consiste en qué tan capaz se siente un individuo para emprender). El instrumento hace uso de una escala tipo Likert de cinco niveles de conformidad (Sullivan et al., 2013).

\section{Recolección de la información}

El instrumento descrito fue aplicado al conjunto de la población de la Universidad Tecnológica El Retońo, integrada por un total de 522 alumnos en el cuatrimestre comprendido entre mayo y agosto del 2019; el rango de edades de los alumnos fluctúa entre los 18 y los 24 ańos, incluyendo individuos que radican en Aguascalientes, Jalisco y Zacatecas, tanto en zonas urbanas como rurales. Las preguntas fueron estructuradas y cerradas, haciendo uso de la herramienta de formularios integrada en la aplicación de Google. Para un mayor control de la información se generó un enlace por cada grupo participante en el estudio, totalizando 29 grupos, 23 del nivel de técnico superior universitario, cinco de nivel Ingeniería y uno de Licenciatura.

\section{Análisis estadístico}

La consistencia interna del instrumento aplicado fue validada a través del alfa de Cronbach (Cronbach, 1951), correlaciones biseriales para evaluar la discriminación de los reactivos, desde la teoría clásica del test (Tate, 2014) y la prueba no paramétrica de correlación de Spearman (Agresti, 2007); las tres pruebas fueron examinadas al interior de cada una de las secciones del mismo. Posteriormente se realizó un análisis de componentes principales (Johnson, 2000), lo mismo para cada una de las cuatro secciones del instrumento, con el propósito de cuantificar la IE de los estudiantes encuestados, la cual sirvió para responder las hipótesis planteadas. Se utilizó el paquete estadístico STATA versión 15 para desarrollar los análisis estadísticos. 


\section{RESULTADOS}

El instrumento de Saadin y Daskin (2015) empleado en este estudio exhibe una apropiada consistencia y discriminación en las respuestas, lo que permite estudiar adecuadamente la IE en los estudiantes de la Universidad Tecnológica El Retońo. Del total de ellos encuestados (522 alumnos), se tuvo una tasa alta de respuesta, ya que 497 fueron respuestas válidas, lo que representa $97 \%$ de la población objeto del estudio, de las cuales, aproximadamente $50 \%$ fueron hombres y $50 \%$ mujeres (gráficas 1 y 2 ).

- Gráfica 1. Distribución de alumnos encuestados de Ingeniería/Licenciatura

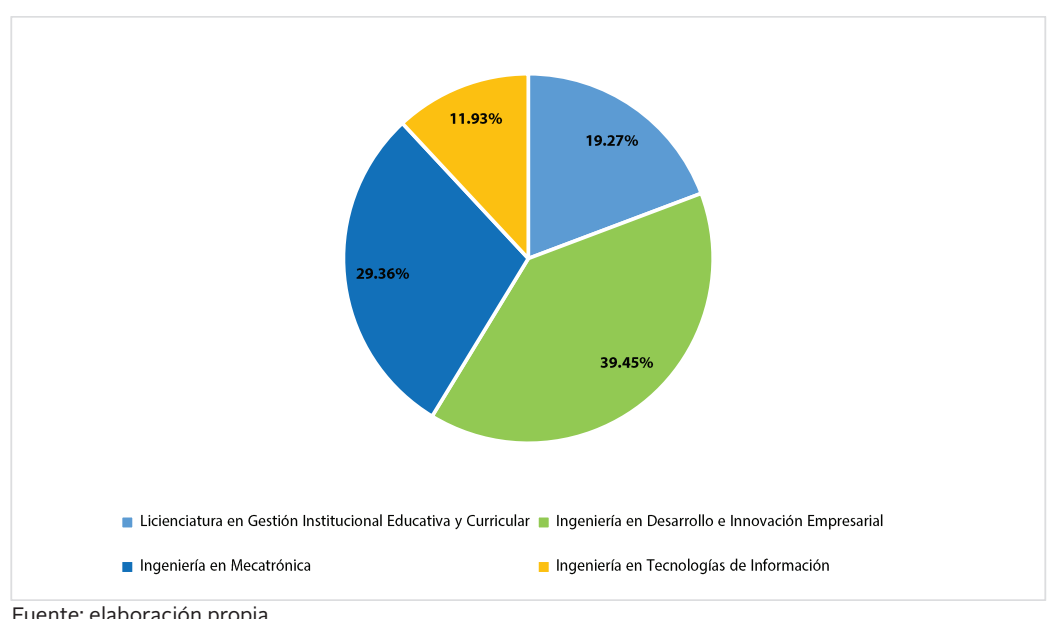

Fuente: elaboración propia.

- Gráfica 2. Distribución de alumnos encuestados de TSU

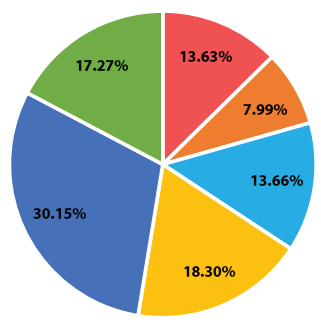

$$
\begin{aligned}
& \text { administración Área Capital Humano = Diseño Digital Área Animación } \\
& \text { - Desarrollo de Negocios Área Mercadotecnia —a Lengua Inglesa } \\
& \text { - Mecatrónica } \\
& \text { - Tecnologías de Información y Comunicación }
\end{aligned}
$$


La consistencia interna del instrumento de Saadin y Daskin empleado en este estudio fue marginal en la sección IV, y muy alta en las secciones I, II y III; se consideraron tres estimadores: alfa de Cronbach, correlaciones biseriales y correlaciones de Spearman, que miden la homogeneidad en los reactivos y, por ende, en sus respuestas, la estrategia fue realizar los análisis para cada una de las cuatro secciones del instrumento, los resultados del alfa de Cronbach y de las correlaciones biseriales se muestran en el cuadro 1 .

De acuerdo con lo establecido por Nunnally y Bernstein (1994), los valores del alfa de Cronbach para medir la consistencia de cada una de las secciones del instrumento aplicado son aceptables debido a que todos están por encima de 0.70 . El análisis de correlaciones biseriales en cada una de las cuatro secciones del instrumento muestra una alta discriminación de los reactivos de cada sección con respecto al total de respuestas en la sección correspondiente. Los resultados de dichas correlaciones fueron obtenidos usando el comando somersd de Stata, cabe mencionar que los valores son muy altos para las secciones I, II y III, a diferencia de los de la cuarta sección, donde su valor es marginal. Todos los resultados son estadísticamente significativos $(\mathrm{p}<0.05)$.

Para medir la intensidad de la correlación de las variables por sección fue utilizada la prueba estadística no paramétrica de Spearman, la cual permite un adecuado tratamiento a la escala de Likert. Saadin y Daskin (2015) presentan un análisis de correlaciones, pero no especifican el método de correlación empleado. Los resultados de la correlación de las variables se muestran mediante el uso de diagramas de color, los cuales se presentan en los diagramas 1, 2, 3 y 4 . 
- Cuadro 1. Coeficientes de la Correlación Biserial y alfa de Cronbach

\begin{tabular}{|c|c|c|c|}
\hline VARIABLE & ELEMENTOS DEL INSTRUMENTO & $\begin{array}{c}\text { Correlación } \\
\text { Biserial }\end{array}$ & $\begin{array}{l}\text { Alfa de } \\
\text { Cronbach }\end{array}$ \\
\hline & INTENCIÓN EMPRENDEDORA & & 0.93 \\
\hline VAL_1 & Probablemente tendré un negocio algún día & 0.58 & \\
\hline VAL_2 & Soy capaz de tomar decisiones que tendrán éxito en mi negocio & 0.43 & \\
\hline VAL_3 & Haré todos los esfuerzos necesarios para abrir mi negocio & 0.59 & \\
\hline VAL_4 & Me arriesgaré a abrir un negocio porque quiero ser mi propio jefe & 0.60 & \\
\hline VAL_5 & Me veo a mí mismo como un empresario exitoso & 0.55 & \\
\hline VAL_6 & Tengo el plan de abrir un negocio en el futuro cercano & 0.63 & \\
\hline VAL_7 & Mi objetivo es convertirme en emprendedor & 0.71 & \\
\hline VAL_8 & Haré lo que sea necesario para abrir un negocio & 0.73 & \\
\hline VAL_9 & Estoy listo para hacer lo que sea para convertirme en emprendedor & 0.69 & \\
\hline \multirow[t]{2}{*}{ VAL_10 } & Mi personalidad refleja la de un emprendedor & 0.53 & \\
\hline & DESEABILIDAD PERCIBIDA & & 0.76 \\
\hline VAL_11 & Prefiero un negocio a una carrera profesional prometedora & 0.47 & \\
\hline VAL_12 & El éxito futuro radica en abrir mi propio negocio & 0.52 & \\
\hline VAL_13 & A mis amigos les gustaría verme iniciar mi propio negocio & 0.56 & \\
\hline VAL_14 & A mis parientes les gustaría verme abrir mi propio negocio & 0.69 & \\
\hline \multirow[t]{2}{*}{ VAL_15 } & $\begin{array}{l}\text { A mi familia directa (padres y hermanos) le gustaría verme abrir mi } \\
\text { propio negocio }\end{array}$ & 0.63 & \\
\hline & NORMAS SOCIALES & & 0.81 \\
\hline VAL_16 & El poseer un negocio es apreciado por la comunidad & 0.59 & \\
\hline VAL_17 & $\begin{array}{l}\text { El poseer un negocio es apreciado por la familia directa (padres y } \\
\text { hermanos) }\end{array}$ & 0.71 & \\
\hline VAL_18 & El poseer un negocio es apreciado por los parientes & 0.77 & \\
\hline \multirow[t]{2}{*}{ VAL_19 } & El poseer un negocio es apreciado por los amigos & 0.67 & \\
\hline & FACTIBILIDAD PERCIBIDA & & 0.80 \\
\hline VAL_20 & Yo puedo administrar un equipo & 0.16 & \\
\hline VAL_21 & Mis habilidades y capacidades me ayudan para abrir un negocio & 0.15 & \\
\hline VAL_22 & Confío en mí para iniciar un negocio & 0.19 & \\
\hline VAL_23 & Tengo acceso a información para convertirme en emprendedor & 0.15 & \\
\hline VAL_24 & $\begin{array}{l}\text { Tengo una buena red de contactos para convertirme en } \\
\text { emprendedor }\end{array}$ & 0.17 & \\
\hline VAL_25 & Es fácil abrir un negocio & 0.14 & \\
\hline
\end{tabular}

Fuente: elaboración propia. 
Diagrama 1. Análisis de Correlación de Spearman de Intención Emprendedora

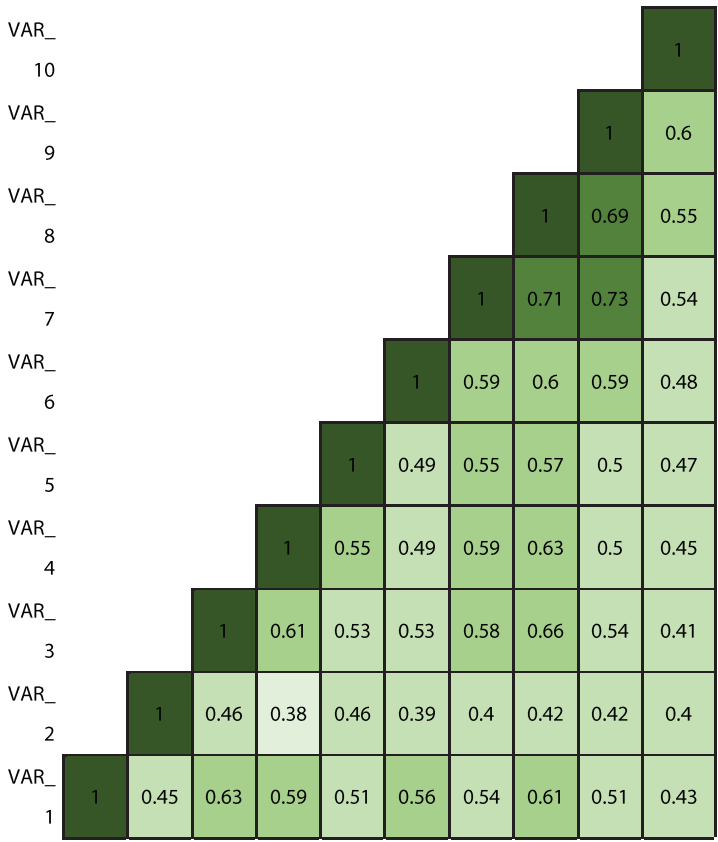

$V A R_{-} V A R_{-} V A R_{-} V A R_{-} V A R_{-} V A R_{-} V A R_{-} V A R_{-} V A R_{-} V A R_{-}$

$\begin{array}{llllllllll}1 & 2 & 3 & 4 & 5 & 6 & 7 & 8 & 9 & 10\end{array}$

Fuente: elaboración propia.

Diagrama 2. Análisis de Correlación de Spearman de Deseabilidad Percibida

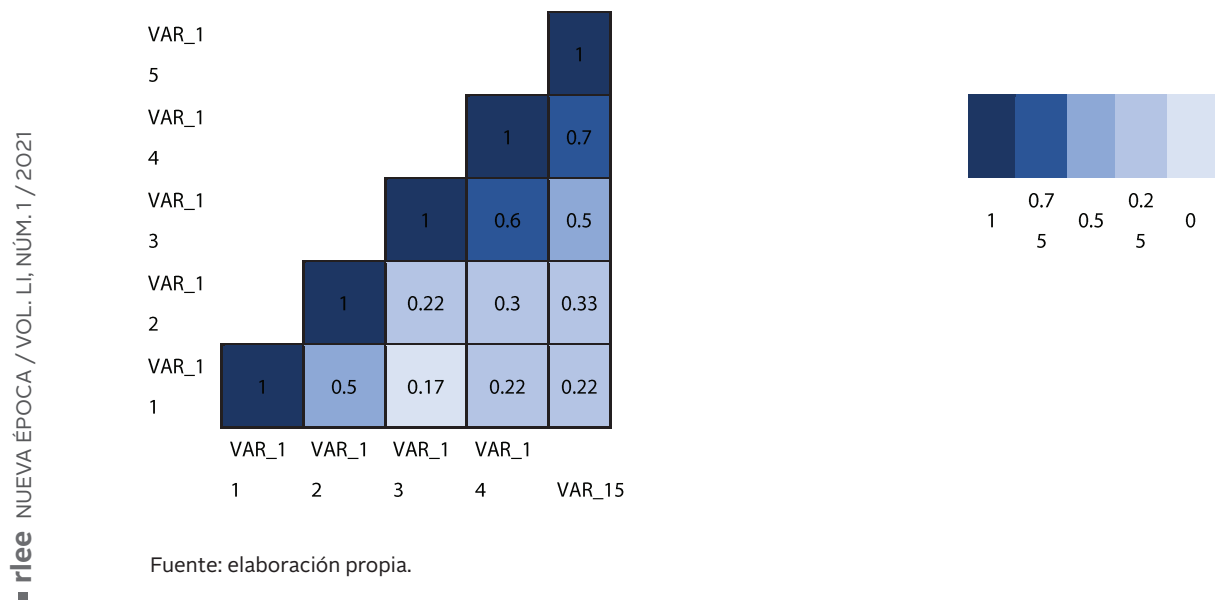


Diagrama 3 . Análisis de Correlación de Spearman de Normas Sociales
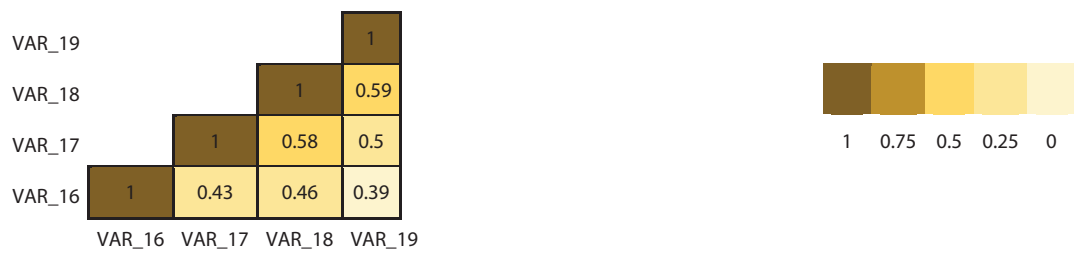

Fuente: elaboración propia.

Diagrama 4. Análisis de Correlación de Spearman de Factibilidad Percibida

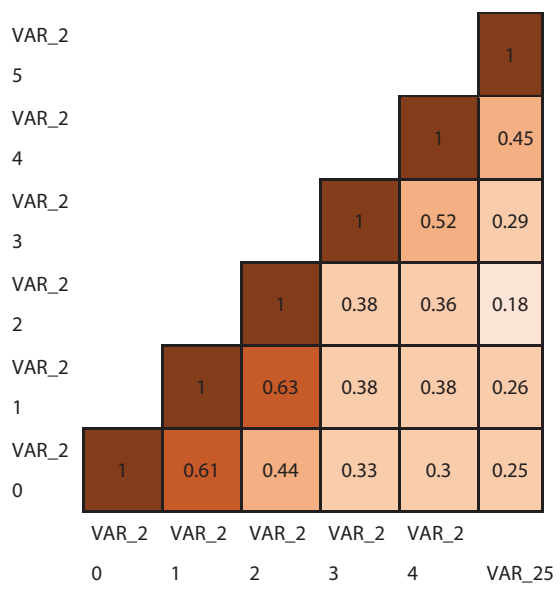

Fuente: elaboración propia.

En el diagrama 1 se aprecia que la variable 8 (VAR_8), la cual se asocia con la apertura de un negocio, es la que más se correlaciona con las variables de la sección de intención emprendedora. En los diagramas 2 y 3 , se observan correlaciones intermedias, lo cual se encuentra en concordancia con el alfa de Cronbach estimado para estas secciones. En el diagrama 4, analizando los coeficientes de correlación de Spearman, se identifica una correlación muy heterogénea entre los reactivos de esa sección. Los resultados presentados en los diagramas 1-4 fueron estadísticamente significativos $(\mathrm{p}<0.05)$.

En concordancia con el propósito del estudio, el cual es medir la IE de forma integral, se utilizó la técnica estadística multivariada de análisis de componentes principales (ACP), la cual es ampliamente recomendada para reducir la dimensión de un problema complejo como el abordado en este trabajo (Rencher, 1998). 
El ACP permitió la determinación de la importancia de cada variable dentro de las cuatro secciones y con ello fue posible la construcción de un índice para medir la IE, el cual se transformó en una escala de 0 a 1 para su mejor comprensión e interpretación. Siguiendo la recomendación de Rencher (1998), no fue utilizada alguna técnica de rotación, dado que los resultados (valores propios) tienen una interpretación acorde con el problema estudiado; es decir, el ACP funciona correctamente, sin necesidad de transformaciones; lo anterior puede explicarse por la homogeneidad de respuestas que se obtienen al utilizar la escala de Likert y la oportunidad de utilizar la matriz de varianzas y covarianzas en lugar de una matriz de correlaciones.

En el cuadro 2 se presenta el porcentaje de varianza explicada y las comunalidades para cada componente, así como los eigenvalores o valores propios y los pesos de importancia de cada variable, los cuales fueron calculados considerando el número de componentes seleccionados, su proporción de valor y su eigenvalor correspondiente. La selección de componentes fue con base en el alfa de Cronbach y las correlaciones biseriales, por ejemplo, en la sección I, se obtuvo un Alfa de Cronbach muy alto (0.93) y correlaciones biseriales muy aceptables (1.00-0.05); por ello se tomó la decisión de sólo incluir el primer componente. Para las secciones II y III fueron incluidos dos componentes y para la sección IV se incluyen los primeros tres componentes, buscando con ello captar de mejor forma la importancia de las variables de esta sección, que tienen marginal discriminación y consistencia de acuerdo con el análisis de correlaciones biseriales. Por último, la columna peso nos informa la importancia de cada una de las variables por sección, considerando el número de componentes seleccionados y para el caso de dos o tres componentes seleccionados se pondera la importancia usando la proporción de varianza explicada por el componente correspondiente; por ejemplo, la importancia de la variable 11 (VAR_11) es Peso $0.37 * 0.52+0.69^{*} .024=0.35$, y así sucesivamente.

En el ACP de la sección I se encontró que la característica con mayor peso (0.38) para la IE de un alumno es tener el objetivo de ser emprendedor. La concordancia de las preguntas en esta sección es muy alta (alfa de Cronbach $=0.93$ ) y las correlaciones biseriales exhiben una discriminación muy aceptable de los reactivos, lo que da mayor validez a los resultados obtenidos con ACP. 
- Cuadro 2. Análisis de Componentes Principales por sección

\begin{tabular}{|c|c|c|c|c|c|c|c|c|c|}
\hline \multirow{2}{*}{ CATEGoGÍA } & \multirow{2}{*}{ VARIABLES } & \multirow{2}{*}{$\begin{array}{c}\text { EIGENVALORES } \\
\text { (VALORES PROPIOS) }\end{array}$} & \multirow{2}{*}{$\begin{array}{l}\text { PROPORCIÓN } \\
\text { DE LA VARIANZA }\end{array}$} & \multirow{2}{*}{$\begin{array}{c}\text { PROPORCIÓN } \\
\text { ACUMULADA DE } \\
\text { VARIANZA }\end{array}$} & \multirow{2}{*}{$\begin{array}{l}\text { NÚMERO DE } \\
\text { COMPONENTES } \\
\text { ELEGIDOS }\end{array}$} & \multicolumn{3}{|c|}{$\begin{array}{l}\text { VALORES PROPIOS PARA } \\
\text { VARIABLES }\end{array}$} & \multirow[t]{2}{*}{ PESOS } \\
\hline & & & & & & $\begin{array}{c}\text { COMP. } \\
1\end{array}$ & $\begin{array}{c}\text { COMP. } \\
2\end{array}$ & $\begin{array}{c}\text { COMP. } \\
3\end{array}$ & \\
\hline \multirow{10}{*}{$\begin{array}{l}\text { INTENCIÓN } \\
\text { EMPRENDEDORA }\end{array}$} & VAL_1 & 4.72 & 0.61 & 0.61 & 1 & 0.28 & & & 0.28 \\
\hline & VAL_2 & 0.59 & 0.08 & 0.69 & & 0.22 & & & 0.22 \\
\hline & VAL_3 & 0.45 & 0.06 & 0.74 & & 0.30 & & & 0.30 \\
\hline & VAL_4 & 0.42 & 0.05 & 0.80 & & 0.31 & & & 0.31 \\
\hline & VAL_5 & 0.34 & 0.04 & 0.84 & & & 0.30 & & 0.30 \\
\hline & VAL_6 & 0.32 & 0.04 & 0.88 & & 0.35 & & & 0.35 \\
\hline & VAL_7 & 0.27 & 0.03 & 0.92 & & 0.38 & & & 0.38 \\
\hline & VAL_8 & 0.22 & 0.03 & 0.95 & & 0.35 & & & 0.35 \\
\hline & VAL_9 & 0.22 & 0.03 & 0.98 & & 0.35 & & & 0.35 \\
\hline & VAL_10 & 0.18 & 0.02 & 1.00 & & 0.29 & & & 0.29 \\
\hline \multirow{5}{*}{$\begin{array}{l}\text { DESEABILIDAD } \\
\text { PERCIBIDA }\end{array}$} & VAL_11 & 2.18 & 0.52 & 0.52 & 2 & 0.37 & 0.69 & & 0.35 \\
\hline & VAL_12 & 1.04 & 0.24 & 0.76 & & 0.40 & 0.48 & & 0.32 \\
\hline & VAL_13 & 0.43 & 0.10 & 0.86 & & 0.44 & -0.32 & & 0.15 \\
\hline & VAL_14 & 0.37 & 0.09 & 0.95 & & 0.51 & -0.33 & & 0.18 \\
\hline & VAL_15 & 0.22 & 0.05 & 1.00 & & 0.50 & -0.27 & & 0.19 \\
\hline \multirow{4}{*}{$\begin{array}{l}\text { NORMAS } \\
\text { SOCIALES }\end{array}$} & VAL_16 & 1.67 & 0.64 & 0.64 & 2 & 0.43 & 0.90 & & 0.41 \\
\hline & VAL_17 & 0.38 & 0.15 & 0.78 & & 0.53 & -0.17 & & 0.31 \\
\hline & VAL_18 & 0.31 & 0.12 & 0.90 & & 0.55 & -0.28 & & 0.31 \\
\hline & VAL_19 & 0.26 & 0.10 & 1.00 & & 0.48 & -0.30 & & 0.26 \\
\hline \multirow{6}{*}{$\begin{array}{l}\text { FACTIBILIDAD } \\
\text { PERCIBIDA }\end{array}$} & VAL_20 & 2.52 & 0.51 & 0.51 & 3 & 0.39 & 0.51 & 0.20 & 0.29 \\
\hline & VAL_21 & 0.88 & 0.18 & 0.68 & & 0.38 & 0.43 & & 0.27 \\
\hline & VAL_22 & 0.58 & 0.12 & 0.80 & & 0.34 & 0.36 & & 0.24 \\
\hline & VAL_23 & 0.44 & 0.09 & 0.89 & & 0.43 & -0.24 & -0.62 & 0.17 \\
\hline & VAL_24 & 0.38 & 0.08 & 0.96 & & 0.54 & -0.46 & -0.11 & 0.19 \\
\hline & VAL_25 & 0.18 & 0.04 & 1.00 & & 0.34 & -0.41 & 0.75 & 0.10 \\
\hline
\end{tabular}

Fuente: elaboración propia.

El análisis de correlación mostrado en el diagrama 2 exhibe correlaciones bajas entre la mayoría de las variables de esta sección; se determina que la característica de los alumnos relacionada con su preferencia a emprender un negocio es la que mayor importancia tiene para medir la deseabilidad percibida. Este ACP permite apre- 
ciar la conformación de dos grupos, uno que reúne lo relacionado a negocios y otro con la percepción de amigos y familiares, la importancia de emprender un negocio es mayor que la importancia que dan a la percepción de amigos y familiares.

En lo que corresponde a la sección III de normas sociales, tanto el análisis de correlación del diagrama 3 y ACP el cuadro 2 exhiben correlaciones y pesos intermedios, los cuales permiten la identificación de características similares.

En su mayoría, las correlaciones de las características de la sección IV de factibilidad percibida del diagrama 4 muestran valores intermedios y, con ello, no es factible la determinación de patrones de agrupamiento. Sin embargo, el ACP nos permite identificar que la conformación de una buena red de contactos fomenta la factibilidad percibida por parte de los alumnos para el emprendedurismo.

Empleando el índice de IE y utilizando una prueba t de comparación de medias, se contrastan las hipótesis 1 y 2. En la hipótesis 1 fue analizada la media de IE para los alumnos que cursan la carrera de administración a nivel ingeniería (últimos cuatrimestres) versus los alumnos que están inscritos en el programa de Técnico Superior (primeros cuatrimestres), la diferencia de medias cuenta el efecto y se incluyen los resultados en el cuadro 3.

- Cuadro 3. Comparación de medias del IE por grado

\begin{tabular}{|l|c|c|c|c|c|}
\hline $\begin{array}{c}\text { NIVEL } \\
\text { ACADÉMICO }\end{array}$ & OBSERVACIONES & PROMEDIO & DESVIACIÓN ESTÁNDAR & \multicolumn{2}{|c|}{ INTERVALO AL 95\% DE CONFIANZA } \\
\hline ING/LIC & 109 & 0.72 & 0.13 & 0.70 & 0.75 \\
\hline TSU & 388 & 0.68 & 0.14 & 0.67 & 0.70 \\
\hline
\end{tabular}

Fuente: elaboración propia.

En cuanto a la primera hipótesis planteada, referente a que los alumnos que están cursando los últimos cuatrimestres tienen IE más alta que el resto de los estudiantes, se encontró: 1) los alumnos que cursan ingeniería o licenciatura tienen IE más alta que los alumnos de TSU; 2) analizado por carrera, los alumnos de la Ingeniería en Desarrollo e Innovación Empresarial tienen la IE más alta, seguidos por los de Ingeniería en Mecatrónica y Licenciatura en Gestión Ins- 
titucional Educativa y Curricular; por otro lado, los alumnos que presentan la IE más baja son los de Ingeniería en Tecnologías de la Información; 3) considerando a los alumnos por grado y grupo, los alumnos de uno de los dos grupos de la Licenciatura en Gestión Institucional Educativa y Curricular tienen la más alta IE; 4) en cuanto a las carreras tecnológicas, la que presenta una IE más alta es la Ingeniería en Mecatrónica, le sigue el TSU en Mecatrónica, siendo la que presenta la IE más baja la Ingeniería en Tecnologías de la Información (la IE que presenta es más baja, incluso que la del TSU de esa misma carrera); 5) en cuanto al área de Diseño digital, los alumnos que presentan la IE más alta son los de los primeros cuatrimestres; 6) los alumnos del área de idioma Inglés, en particular los que cursan los últimos cuatrimestres (Licenciatura en Gestión Institucional Educativa y Curricular), presentan la IE más alta que los alumnos de TSU (Lengua Inglesa).

Para el caso de la segunda hipótesis se construyen dos grupos: uno para aquellas con enfoque administrativa y el complemento conformó el segundo grupo, usando una prueba t fueron comparadas las medias de IE y medido el efecto, el resultado de este análisis se muestra en el cuadro 4.

- Cuadro 4. Comparación de medias de IE por tipos de carrera

\begin{tabular}{|c|c|c|c|c|c|}
\hline TIPO DE CARRERAS & OBSERVACIONES & PROMEDIO & DESVIACIÓN ESTÁNDAR & \multicolumn{2}{|c|}{ INTERVALO AL 95\% DE CONFIANZA } \\
\hline ADMINISTRATIVAS & 145 & 0.74 & 0.12 & 0.72 & 0.76 \\
\hline NO ADMINISTRATIVAS & 352 & 0.67 & 0.14 & 0.66 & 0.69 \\
\hline
\end{tabular}

Fuente: elaboración propia.

La segunda hipótesis planteada se refiere a que los estudiantes de las carreras administrativas tienen la IE más alta. Al respecto se encontró lo siguiente: 1) analizando todas las carreras administativas tanto de TSU como de ingeniería, la IE más alta es para los alumnos de la Ingeniería en Desarrollo e Innovación Empresarial; le siguen los alumnos del TSU en Desarrollo de Negocios Área Mercadotecnia y, al último, se encuentran los alumnos de Administración Área Capital Humano, lo que destaca lo planteado en la hipótesis 1, por ser 
los alumnos de los últimos cuatrimestres los que poseen una IE más alta; 2) en el cuatrimestre mayo-agosto 2019, se tenían dos grupos para segundo cuatrimestre de la carrera Administración Área Capital Humano y Desarrollo de Negocios Área Mercadotecnia, y dos grupos de quinto cuatrimestre para las mismas carreras, además de contar con dos grupos para la Ingeniería en Desarrollo e Innovación Empresarial, cabe resaltar que la IE más alta fue para uno de los grupos de Ingeniería; sin embargo, el grupo que le siguió fue de segundo cuatrimestre de Administación Área Capital Humano (gráfica 3).

Gráfica 3. Valores del Índice de Intención Emprendedora en los alumnos de Ingeniería/Licenciatura y TSU

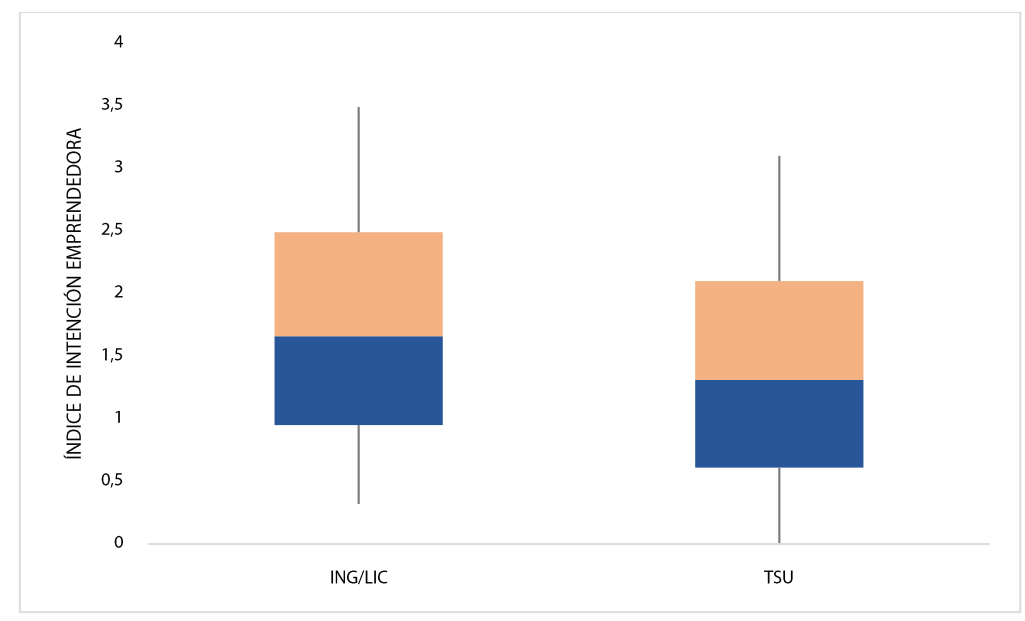

Fuente: elaboración propia.

\section{DISCUSIÓN}

Se ha estudiado que algunas variables tienen efectos positivos en el desarrollo de la IE, entre las cuales se encuentran: 1) la percepción de las habilidades (autoeficacia) (Vidal-Suñé y López-Panisello, 2013), influida ésta por la inteligencia emocional, la capacidad para regular y usar adecuadamente las emociones (Mortan et al., 2014); 2) el alto nivel de regulaciones como protección de la incertidumbre (Vidal-Suñé et al., 2013); 3) tener estudios universitarios (Zampetakis, 2008, Romero-Martínez y Milone, 2016, Martínez-Gámez, 
2016, Potishuk y Kratzer, 2017); 4) la educación o capacitación de emprendimiento (Soria-Barreto et al., 2017), preferentemente con grupos interdisciplinarios (Padilla-Angulo et al., 2019); 5) la capacidad para solucionar problemas (Soria-Barreto et al., 2016a) y la alta creatividad y proactividad de los individuos (Zampetakis, 2008); 6) la renta familiar baja (Soria-Barreto et al., 2016b, Martínez-Gámez, 2016); 7) el incentivar el emprendimiento durante la adolescencia (Obschonka et al., 2010, Soria-Barreto et al., 2017); 8) la insatisfacción laboral (Martínez-Gámez, 2016); 9) la adquisición de recursos u oportunidades tangibles (p. e. capital para invertir) o intangibles (habilidad de establecer redes sociales, el talento para comercializar un producto, etc.) (Zhang et al., 2015).

Las variables que tienen efectos negativos en la IE, son: 1) el incremento en el Producto Interno Bruto (Vidal-Suñé y López-Panisello, 2013); 2) el riesgo (Torres et al., 2018, y Soria-Barreto et al., 2016b), porque impacta en la motivación de lograr una meta y en el control percibido; 3 ) el actuar en condiciones de incertidumbre (Soria-Barreto et al., 2016b); 4) el tener una economía en vías de desarrollo y que existan altos niveles de competitividad en las empresas (GEM, 2018).

Por estar influida la IE de tantas formas y con tan diferentes resultados, un primer paso ya se dio al determinar el índice de IE de los alumnos de la comunidad de la Universidad Tecnológica El Retońo para seguir determinando los factores que influyen positiva y negativamente en cada individuo.

\section{CONCLUSIONES}

Este trabajo analiza la discriminación del instrumento propuesto por Saadin y Daskin (2015) para la población de alumnos de la Universidad Tecnológica El Retońo usando tres enfoques: 1) estadística no paramétrica con la matriz de correlaciones de Spearman, 2) la teoría clásica de los ítems con las correlaciones biseriales y 3) el enfoque multivariado de ACP. La contribución es la implementación de una metodología de construcción de un índice de emprendimiento que permitirá a la institución medir y, posteriormente, evaluar la evolución de la política de fortalecimiento de la intención de emprendimiento en los alumnos 
Un resultado de este estudio es que se detecta que la sección IV del instrumento aplicado, correspondiente a la factibilidad percibida, presenta una discriminación marginal para el estudio de la intención de emprendimiento y por ello esta sección debe de reformularse y posteriormente validarse usando una población de ensayo y prueba. Lo anterior se fundamenta en el análisis de correlaciones biserial, no parámetrico de Spearman y el análisis de componentes principales.

La metodología estadística de análisis de componentes principales permitió distinguir de manera integral y robusta las múltiples variables que intervienen en la IE, sintetizándolas en un índice. Se concluye que los alumnos de los últimos cuatrimestres poseen la IE más alta de la institución; es decir, la IE de los alumnos de Ingeniería o Licenciatura es mayor que la de los alumnos que cursan TSU, con la única excepción del TSU de Tecnologías de Información y Comunicación, cuya IE es superior a la de los alumnos que cursan la Ingeniería en Tecnologías de Información. En todos los casos, se tendrá que seguir de cerca el desarrollo de los alumnos de los primeros cuatrimestres de cada carrera, para comprobar la afectación (positiva o negativa) conforme cursen el resto de las asignaturas de su programa educativo.

Otro hallazgo fue que los alumnos de TSU presentan una mayor variabilidad en los resultados, lo cual indica una falta en el rumbo del emprendimiento, y también afecta en su IE, debido a esto los alumnos de ingeniería y licenciatura poseen una IE más alta. Como objeto de estudios posteriores será necesario analizar los datos atípicos, para determinar las características de esos alumnos y poder ofrecerles alternativas para que fortalezcan e incrementen su IE.

Los alumnos que cursan carreras administrativas presentan el más alto índice de IE, es decir la Ingeniería en Desarrollo e Innovación empresarial sobresale del resto de las carreras; en cuanto a TSU, la carrera de Desarrollo de Negocios Área Mercadotecnia encabeza la lista. En ambos casos, 49\% de los estudiantes presenta una escala Alta en el índice de IE. Sin embargo, cabe hacer mención que la carrera de TSU de Administración Área Capital Humano presenta números similares a los de la carrera de Diseño Digital Área Animación, lo cual pone en duda lo planteado en el estudio en cuanto a que cursar asignaturas en el área del emprendimiento asegura un incremento en la IE, lo cual 
da pie a nuevas investigaciones para observar otras variables que estén impactando en el emprendimiento de los alumnos, como podrían ser: antecedentes académicos y familiares y otras influencias que han tenido los alumnos a lo largo de sus vidas.

Se deberá observar y medir la IE de los alumnos considerando su trayectoria dentro de la universidad y, posteriormente, medir su desempeño como egresados, considerando que la intención no necesariamente genera acción y que tendrán que pasar varios años para corroborar que los alumnos de la Universidad Tecnológica El Retoño que presentan una mayor IE realmente la materialicen creando empresas o bien como intraemprendedores (emprendimientos dentro de las empresas). Esto sugiere un estudio longitudinal en el que se analicen las variables que pudieran impactar en dicho proceso.

El reforzamiento de las asignaturas y de programas alternos de emprendimiento son trascendentes para que no sólo alumnos de áreas administrativas tengan dicha formación. Se sugiere trabajar en particular con los alumnos de las áreas tecnológicas, para ofrecerles posibilidades de desarrollar innovación desde el lado del emprendimiento, y concientizar a toda la comunidad universitaria para aportar su saber en trabajos interdisciplinarios, para conformar start ups que favorezcan el desarrollo tecnológico de la región.

\section{REFERENCIAS}

Agresti, A. (2007). An Introduction to Categorical Data Analysis. Estados Unidos: Wiley-Interscience.

Ajzen, I. (1991). The theory of planned behavior. Organizational Behavior and Human Decision Processes, 50(2), 179-211.

Bird, B. (1988). Implementing Entrepreneurial Ideas: The Case for Intention. The Academy of Management Review, 13(3), 442-453.

Contreras, F., Espinosa, J. C., Soria, K., Portalanza, A., Jáuregui Machuca, K., y Omańa, J. A. (2017). Exploring entrepreneurial intentions in Latin American university students. International Journal of Psychological Research, 10(2), 46-59. https://doi.org/10.21500/20112084.2794

Cronbach, L. J. (1951). Coefficient alpha and the internal structure of tests* If $\sim$ j. cronbach. Psychometrika, 16(3), 297-298.

Dugassa, T. G. (2012). Impact of entrepreneurship education on entrepreneurial intentions of business and engineering students in Ethiopia. 
African Journal of Economic and Management Studies, 3(2), 258-277. https://doi.org/10.1108/20400701211265036

Fayolle, A. (2013). Personal views on the future of entrepreneurship education. Entrepreneurship and Regional Development, 25(7-8), 692-701. https://doi.org/10.1080/08985626.2013.821318

Fayolle, A., y Liñán, F. (2014). The future of research on entrepreneurial intentions. Journal of Business Research, 67(5), 663-666. https://doi. org/10.1016/j.jbusres.2013.11.024

GEM (2018). Global Entrepreneurship Monitor 2017/2018. En Global Entrepreneurship Monitor. http://www.gemconsortium.org/report $/ 50012$

Hansemark, O. C. (1998). The effects of an entrepreneurship programme on Need for Achievement and Locus of Control of reinforcement. Revista Internacional de Investigación y Comportamiento Empresarial, $4(1), 28-50$.

Johnson, D. E. (2000). Applied multivariate methods for data analysts.

Kautonen, T., van Gelderen, M., y Fink, M. (2015). Robustness of the theory of planned behavior in predicting entrepreneurial intentions and actions. Entrepreneurship: Theory and Practice, 39(3), 655-674. https://doi.org/10.1111/etap.12056

Krueger, N. F., Reilly, M. D., y Carsrud, A. L. (2000). Competing models of entrepreneurial intentions. Journal of Business Venturing, 15(5-6), 411-432. https://doi.org/10.1016/S0883-9026(98)00033-0

Martínez-Gámez, Á. E. (2016). Factores socioculturales asociados al emprendedor: evidencia empírica para América Latina. Revista Venezolana de Gerencia, 21(74), 312-330.

Mayer, E. L., Charles, J. A., y De la Garza, M. I. (2019). El fomento emprendedor desde la universidad mexicana. Revista Venezolana de Gerencia, 24(85), 49-67. http://www.produccioncientificaluz.org/index. $\mathrm{php} / \mathrm{rvg} /$ article/view/23828

Mortan, R., Ripoll, P., Carvalho, C., y Bernal, M. C. (2014). Effects of emotional intelligence on entrepreneurial intention and self-efficacy. Journal of Work and Organizational Psychology, 29(2), 45-50. https:// doi.org/10.5093/tr2013a7.

Nunnally, J., y Bernstein, I. (1994). Book Review: Psychometric theory (3rd ed.). Journal of Psychoeducational Assessment, 17(3), 27-280. https://doi.org/10.1177/073428299901700307 
Obschonka, M., Silbereisen, R. K., y Schmitt-Rodermund, E. (2010). Entrepreneurial intention as developmental outcome. Journal of Vocational Behavior, 77(1), 63-72. https://doi.org/10.1016/j.jvb.2010.02.008 Padilla-Angulo, L., Díaz-Pichardo, R., Sánchez-Medina, P., y Ramboarison-Lalao, L. (2019). Classroom interdisciplinary diversity and entrepreneurial intentions. Education + Training, 61(7/8), 832-849. https://doi.org/10.1108/ET-06-2018-0136

Potishuk, V., y Kratzer, J. (2017). Factors Affecting Entrepreneurial Intentions and Entrepreneurial Attitudes in Higher Education. Journal of Entrepreneurship Education, 20(1). https://www.abacademies.org/ abstract/factors-affecting-entrepreneurial-intentions-and-entrepreneurial-attitudes-in-higher-education-6571.html

Raposo, M., y Paço, A. (2011). Entrepreneurship education: Relationship between education. Psicothema, 23(3), 453-457.

Rencher, A. C. (1998). Methods of Multivariate Analysis. Journal of the American Statistical Association, 93(443), 1239-1239. https://doi. org/10.2307/2669873

Romero-Martínez, A. M., y Milone, M. (2016). El emprendimiento en España: Intención emprendedora, motivaciones y obstáculos. Journal Globalization, Competitiveness and Governability, 10(1), 95-109. https://doi.org/10.3232/GCG.2016.V10.N1.05

Saadin, M. N., y Daskin, M. (2015). Perceived desirability, feasibility, and social norms as antecedents on hospitality students' entrepreneurial intention in Malaysia: Does gender make a difference? International Journal of Entrepreneurship and Small Business, 25(4), 456-474. https://doi.org/10.1504/IJESB.2015.070218

Shapero, A., y Sokol, L. (1982). The social dimensions of entrepreneurship. En C. Kent, D. L. Sexton y K. H. Vesper (eds.), Encyclopedia of Entrepreneurship (pp. 72-90). Englewood Cliffs, Nueva Jersey: Prentice-Hall. Segal, G., Borgia, D., y Schoenfeld, J. (2005). The motivation to become an entrepreneur. International Journal of Entrepreneurial Behaviour and Research, 11(1), 42-57. https://doi.org/10.1108/13552550510580834 Soria-Barreto, K., Honores-Marin, G., Gutiérrez-Zepeda, P., y GutiérrezRodríguez, J. (2017). Prior exposure and educational environment towards entrepreneurial intention. Journal of Technology Management and Innovation, 12(2), 45-58. https://doi.org/10.4067/S071827242017000200006 
Soria-Barreto, K., Zúñiga-Jara, S., y Ruiz-Campo, S. (2016a). Educación e intención emprendedora en estudiantes universitarios: Un caso de estudio. Formacion Universitaria, 9(1), 25-34. https://doi.org/10.4067/ S0718-50062016000100004

Soria-Barreto, K., Zuñiga-Jara, S., y Ruiz-Campo, S. (2016b). Determinantes de la intención emprendedora: nueva evidencia. Interciencia, 41(5), 325-329

Sullivan, G. M., y Artino, A. R. (2013). Analysing and Interpreting Data From Likert - Type Scales. Journal of Graduate Medical Education, 5(4), 541-542

Tate, R. F. (2014). Applications of Correlation Models for Biserial Data. Journal of American Statistical Association, 50(272), 1078-1095.

Torres, J. A., Valencia, A., Bermúdez, J., Díez-Echavarría, L. F., Urrego, M. L., y Maussa, F. O. (2018). Characterization of entrepreneurial intention in university students as from Systemic Entrepreneurship Intention Model: A case study. Cuadernos de Gestión, 18(2), 95-114. https://doi.org/10.5295/cdg.160670jt

Valencia, A., Cadavid, L., Ríos, D. C., y Awad, G. (2012). Factores que inciden en las intenciones emprendedoras de los estudiantes. Revista Venezolana de Gerencia, 17(57), 132-148. http://www.redalyc.org/articulo.oa?id=29021992008

Vidal-Suñé, A., y López-Panisello, M. B. (2013). Institutional and economic determinants of the perception of opportunities and entrepreneurial intention. Investigaciones Regionales, (26), 75-96.

Zampetakis, L. A. (2008). The role of creativity and proactivity on perceived entrepreneurial desirability. Thinking Skills and Creativity, 3(2), 154-162. https://doi.org/10.1016/j.tsc.2008.07.002

Zhang, P., Wang, D. D., y Owen, C. L. (2015). A study of entrepreneurial intention of university students. Entrepreneurship Research Journal, 5(1), 61-82. https://doi.org/10.1515/erj-2014-0004 